\title{
Inhibition of the Jagged/Notch pathway inhibits retinoblastoma cell proliferation via suppressing the PI3K/Akt, Src, p38MAPK and Wnt/ $\beta$-catenin signaling pathways
}

\author{
WEI XIAO*, XIAOYUN CHEN* ${ }^{*}$ and MINGGUANG HE \\ State Key Laboratory of Ophthalmology, Zhongshan Ophthalmic Center, Sun Yat-sen University, \\ Guangzhou, Guangdong 510060, P.R. China
}

Received July 26, 2013; Accepted March 28, 2014

DOI: $10.3892 / \mathrm{mmr} .2014 .2213$

\begin{abstract}
The Notch signaling pathway is a highly conserved developmental pathway, which is important in the regulation of cellular proliferation, differentiation and apoptosis. The aberrant expression of the Notch pathway has been associated with carcinogenesis in various types of cancer. In order to investigate the expression profiles and biological functions of Notch receptors and ligands in retinoblastoma, the expression levels of their proteins in the human retinoblastoma cell line SO-Rb50 using western blot analysis was assessed. The present study revealed that Notch1 and Jagged 2 were highly expressed compared with human retinal pigment epithelial cells. When treated with DAPT, a specific inhibitor of Notch receptor cleavage, expression of Notch1 and Jagged 2 were downregulated in a dose-dependent manner, which was accompanied by substantial cell growth arrest, as indicated by the Cell Counting kit- 8 assay. In addition, phosphorylation of Akt, p38 mitogen-activated protein kinase and Src, together with the expression of phosphoinositide 3-kinase and $\beta$-catenin, was abated in a dose-dependent manner. However, expression of either total extracellular signal-regulated kinase (Erk)1/2 or phospho-Erk1/2 was not changed in SO-Rb50 cells. These findings demonstrated that the Jagged2/Notch1 pathway can promote oncogenesis in retinoblastoma in co-operation with multiple signaling pathways. The inhibition of the Notch signaling pathway by DAPT represents a potentially attractive strategy for the therapy of retinoblastoma.
\end{abstract}

Correspondence to: Professor Mingguang He, State Key Laboratory of Ophthalmology, Zhongshan Ophthalmic Center, Sun Yat-sen University, 54S Xianlie Road, Guangzhou, Guangdong 510060, P.R. China

E-mail: mingguang_he@yahoo.com

${ }^{*}$ Contributed equally

Key words: Notch signaling pathway, retinoblastoma, DAPT, cell proliferation

\section{Introduction}

Retinoblastoma is the most common intraocular malignancy in children. The incidence of retinoblastoma is constant at 1/15,000-1/20,000 live births worldwide, which corresponds to 29,000 new cases every year (1). If diagnosed early and treated appropriately, children with retinoblastoma are able to be cured. However, the prognosis remains poor in developing countries (2), where mortality due to retinoblastoma is common.

It is well accepted that retinoblastoma is initiated by the mutation of the tumor suppressor gene retinoblastoma 1 (RBI) (3). Numerous studies have suggested that Knudson's M1 and M2 mutational events are necessary for initiating the oncogenesis of retinoblastoma; however, other tumor suppressor genes or oncogenes may also participate in the aggressive progression of this tumor (4). The initiating events are only sufficient for driving the formation of the benign retina tumor, or retinoma; however, they are not enough to lead to the development of malignancy (5). Thus, additional mutational events or other biological behaviors are required for the development from retinoma into retinoblastoma. In addition to its cell biology, there are also numerous unclear aspects associated with the presence of chemoresistance that must be addressed (6). Therefore, the development of novel strategies for the treatment of retinoblastoma is highly desirable for improving the survival outcome of this disease.

Notch signaling is a highly conserved pathway, which mediates signaling between adjacent cells directly in metazoans (7). In mammals, five canonical Notch ligands (Jagged1 and 2 and Delta-like 1, 3 and 4) and four Notch receptors (Notch1-4) have been described to date. Notch receptors are activated by binding to their transmembrane ligands on nearby cells. This interaction further triggers sequential receptor cleavages within the transmembrane domain and the release of the Notch intracellular domain (NICD). Following moving into the nucleus, NICD binds to the transcriptional factor $\mathrm{CBF} / \mathrm{Su}(\mathrm{H}) / \mathrm{LAG}-2$ (CSL; also known as RBP-J in rodents), which is normally suppressed by a corepressor complex. Corepressors are replaced by a coactivating complex containing Mastermind-like protein when NICD associates with CSL. Downstream genes, including Hes-1 and Hey-1, are activated subsequently.

Aberrant expression and dysfunction of the Notch pathway is frequently observed in various types of cancer $(8,9)$. In 
tumorigenesis, the Notch pathway is a double-edged sword (10). It can be an adjudicator of survival versus death, proliferation versus growth arrest or differentiation versus 'stemness'. Despite the progress in delineating the expression profiles and functional derangements of Notch signaling pathways in carcinogenesis during the last decade, its biological significance in retinoblastoma has received little attention, if any.

The present study first examined the expression profiles of Notch ligands and receptors in the human retinoblastoma cell line SO-Rb50, and assessed the effect of $N$-[N-(3,5-difluorophenacetyl)-L-alanyl]-S-phenylglycine $t$-butyl ester (DAPT), a Notch signaling inhibitor, on the proliferation of these cells, as well as its underlying mechanism.

\section{Materials and methods}

Cell culture and treatment. Human retinoblastoma cells (SO-Rb50 cells) were obtained from the Department of Pathology of Zhongshan Ophthalmic Center, Sun Yat-sen University (Guangzhou, Guangdong, China). The cells were cultured in RPMI-1640 medium (Gibco-BRL, Grand Island, NY, USA) supplemented with $10 \%$ fetal bovine serum (Gibco-BRL) at $37^{\circ} \mathrm{C}$ in a humidified atmosphere of $95 \%$ air and $5 \% \mathrm{CO}_{2}$. DAPT (Sigma, St. Louis, MO, USA) was dissolved in dimethyl sulfoxide (DMSO; Sigma) to yield a final concentration equal to $10 \mathrm{mmol} / \mathrm{l}$. Cells were then treated with various concentrations of DAPT $(5,10,30$ and $60 \mu \mathrm{mol} / \mathrm{l})$ or DMSO of the same volume (control).

Cell proliferation analysis. The standard colorimetric Cell Counting kit-8 (CCK-8; Dojindo Laboratories, Kumamoto, Japan) was used for the determination of the number of viable cells in cell proliferation assays. SO-Rb50 cells were seeded with a volume of $200 \mu \mathrm{l}$ cell suspension $(10,000$ cells/well $)$ into 96-well plates. Following treatment with DAPT, the cells were incubated for 24 or $48 \mathrm{~h}$. Following that, $20 \mu \mathrm{l} \mathrm{CCK}-8$ was added to each well and incubated for $2 \mathrm{~h}$ at $37^{\circ} \mathrm{C}$. Optical densities were measured using a microplate reader (Elx800; Bio-Tek Inc., North Brunswick, NJ, USA) by scanning at $450 \mathrm{~nm}$. Cell survival rates were measured at two time-points of the cell growth curve.

Western blot analysis. Cells were collected and lysed with cell lysis buffer. The cell lysates were collected following centrifugation. An equal amount of protein was loaded and separated on $10 \%$ SDS-PAGE. Following being transferred onto polyvinylidene difluoride membranes (Millipore, Bedford, MA, USA), the membranes were inhibited in 5\% skimmed milk for $1 \mathrm{~h}$ and then incubated with primary antibodies at $4{ }^{\circ} \mathrm{C}$ overnight. Following washing three times with phosphate-buffered saline containing $0.1 \%$ Tween-20, the membranes were incubated with horseradish peroxidase (HRP)-conjugated secondary antibodies for $1 \mathrm{~h}$ at room temperature. The membranes were incubated with SignalFire ${ }^{\mathrm{TM}}$ Elite ECL Reagent (Cell Signaling Technology, Beverly, MA, USA) and exposed to $\mathrm{X}$-ray film. $\beta$-actin was used as the loading control. Primary antibodies, including rabbit $\mathrm{mAb}$ anti-Jagged1, rabbit $\mathrm{mAb}$ anti-Jagged2, rabbit $\mathrm{mAb}$ anti-Notch1, rabbit $\mathrm{mAb}$ anti-Notch2, rabbit $\mathrm{mAb}$ anti-Notch3, rabbit $\mathrm{mAb}$ anti-PI3K, rabbit $\mathrm{mAb}$ anti-Akt, rabbit $\mathrm{mAb}$ anti-p-Akt, rabbit mAb anti-p38MAPK, rabbit $m A b$ anti-p-Src, rabbit $m A b$ anti-p-Erk1/2, rabbit $\mathrm{mAb}$ anti- $\beta$-catenin, mouse $\mathrm{mAb}$ anti- $\beta$-actin, mouse $\mathrm{mAb}$ anti-Erk1/2, mouse mAb anti-p-p38MAPK and mouse mAb anti-Src, together with horse anti-mouse and goat anti-rabbit HRP-conjugated secondary antibodies were purchased from Cell Signaling Technology.

Statistical analysis. Each experiment was repeated at least three times. The results are expressed as the mean \pm standard error. Student's t-test was performed for the estimation of statistical significance. $\mathrm{P}<0.05$ was considered to indicate a statistically significant difference.

\section{Results}

Notchl and Jagged2 are highly expressed in $\mathrm{SO}-\mathrm{Rb} 50$ retinoblastoma cells. In order to understand the role of Notch signaling in retinoblastoma, the present study initially sought to identify which Notch receptors and ligands were primarily expressed in SO-Rb50 cells. Western blot analysis was performed to measure the protein expression levels of Notch1, Notch2, Notch3, Jagged1 and Jagged2. Their expression in a human retinal pigment epithelial cell line, ARPE-19, was set as the control. A significantly higher expression of Jagged2 (4.23 \pm 0.35 fold change; $\mathrm{P}<0.05)$ and Notch1 $(11.6 \pm 1.40$ fold change; $\mathrm{P}<0.05)$ was detected in $\mathrm{SO}-\mathrm{Rb} 50$ cells (Fig.1 A and B). The expression levels of Jagged1 in these two types of cell lines were comparable. However, ARPE-19 exhibited much higher expression levels of Notch2 and Notch3, while they were minimal in SO-Rb50 cells. These results suggest that Notch signaling is important in oncogenesis in retinoblastoma.

Inhibition of the Notch pathway by the $\gamma$-secretase inhibitor DAPT is able to downregulate the expression of Jagged 2 and Notchl. To determine the effects of a $\gamma$-secretase inhibitor on the Notch signaling pathway, the protein expression levels of Notch1 and Jagged2 in DAPT-treated SO-Rb50 cells were examined by western blot analysis. The expression of Jagged2 and Notch1 were downregulated following treatment with DAPT for $24 \mathrm{~h}$. This alteration was more profound for Jagged2 levels, which decreased with increasing DAPT concentration in a dose-dependent manner $(\mathrm{P}<0.05$; Fig.1C and D).

Inhibition of the Notch pathway inhibited SO-Rb50 cell growth. To determine whether DAPT is able to inhibit $\mathrm{SO}-\mathrm{Rb} 50$ cell proliferation, its effect on cell growth was examined by the CCK- 8 assay. Cells were treated with various concentrations of DAPT $(0,5,10,30$ or $60 \mu \mathrm{mol} / 1)$. The results demonstrated that DAPT dose-dependently inhibited SO-Rb50 cell growth. The inhibitory effect was significant when SO-Rb50 cells were treated with $60 \mu \mathrm{mol} / 1 \mathrm{DAPT}$ for $24 \mathrm{~h}$ or with 30 or $60 \mu \mathrm{mol} / 1$ DAPT for $48 \mathrm{~h}\left({ }^{*} \mathrm{P}<0.05\right.$, ${ }^{\#} \mathrm{P}<0.01$; Fig. 2A). Nevertheless, the suppression effect was not significantly different between these two doses at either 24 or $48 \mathrm{~h}(\mathrm{P}>0.05)$. Under the phase-contrast microscope, the cell density was found to be markedly reduced when treated with 30 or $60 \mu \mathrm{mol} / 1$ DAPT for $24 \mathrm{~h}$ (Fig. 2B). These results demonstrated that the inhibition of the Notch pathway with DAPT was able to inhibit SO-Rb50 cell proliferation. 
A

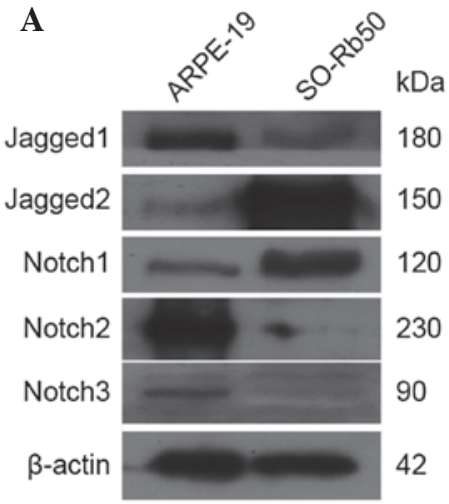

C

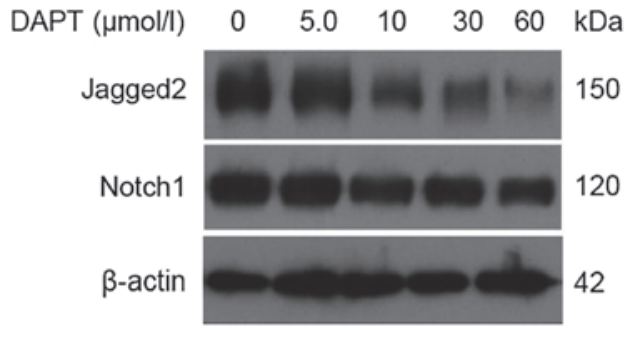

B

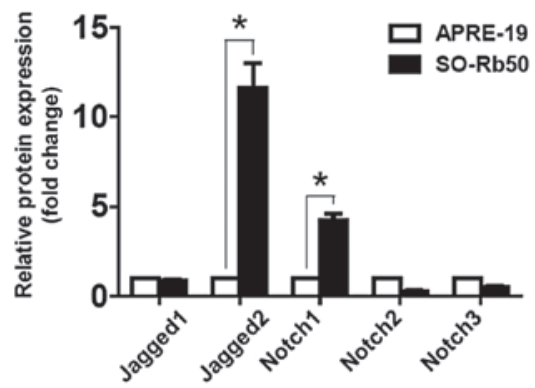

D

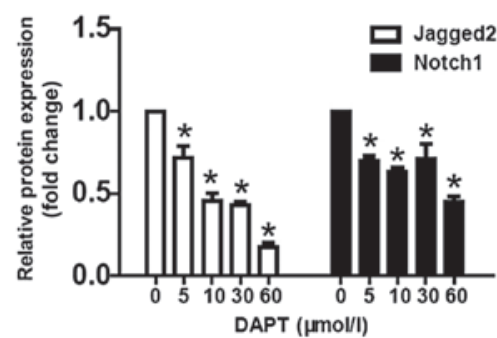

Figure 1. Expression profiles of Notch ligands and receptors in SO-Rb50 cells. Protein expression levels of Jagged1, Jagged2, Notch1, Notch2 and Notch3 were assessed by western blot analysis. Their protein expression in the human retinal pigment epithelial cell line ARPE-19 was used as the control. (A and B) SO-Rb50 cells demonstrated significantly higher expression levels of Notch1 and Jagged2 than ARPE-19 cells. (C and D) When SO-Rb50 cells were treated with various concentrations of DAPT $(5,10,30$ and $60 \mu \mathrm{mol} / \mathrm{l})$, the expression of Notch1 and Jagged 2 was downregulated in a dose-dependent manner. $\beta$-actin was set as the loading control. DAPT, $N$-[ $N$-(3,5-difluorophenacetyl)-L-alanyl]- $S$-phenylglycine $t$-butyl ester. ${ }^{*} \mathrm{P}<0.05$.

A

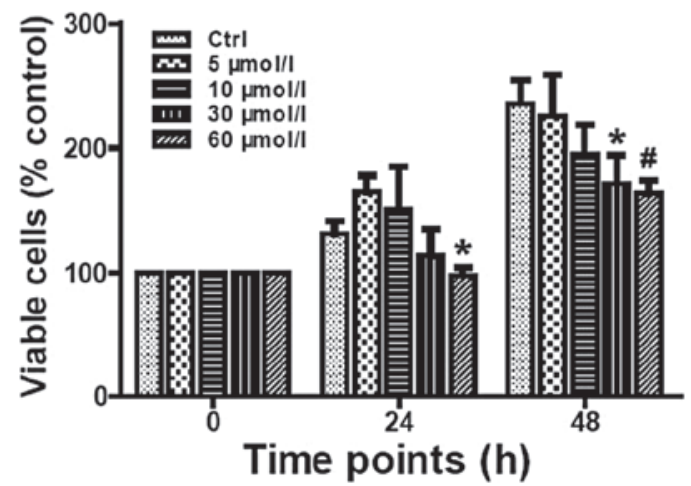

B

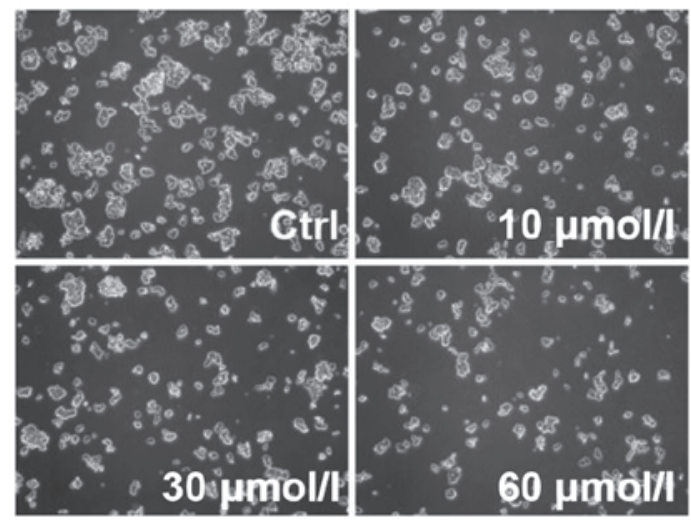

Figure 2. The growth of SO-Rb50 cells was markedly arrested following DAPT treatment. Cell proliferation was assessed using the CCK- 8 assay following 24 or $48 \mathrm{~h}$ treatment with DAPT. (A) The suppressive effect was significant when cells were treated with $60 \mu \mathrm{mol} / 1 \mathrm{DAPT}$ for $24 \mathrm{~h}$ or with 30 or $60 \mu \mathrm{mol} / 1 \mathrm{DAPT}$ for $48 \mathrm{~h}$. (B) When observed under an inverted microscope, the cell density was apparently decreased in DAPT-treated groups (magnification, x100). CCK-8, Cell Counting kit-8; DAPT, $N$-[N-(3,5-difluorophenacetyl)-L-alanyl]-S-phenylglycine $t$-butyl ester. ${ }^{*} \mathrm{P}<0.05 ;{ }^{*} \mathrm{P}<0.01$.

DAPT modulates cell growth through the regulation of the $P I 3 K / A k t, S r c, p 38 M A P K$ and wnt/ $\beta$-catenin pathways; however, it has no effect on the Erk1/2 pathway. The Notch signaling pathway has been demonstrated to crosstalk with the epidermal growth factor receptor pathway (11). Therefore, several key signaling pathways associated with cell growth, including PI3K/Akt, Src, p38MAPK, wnt/ $\beta$-catenin and Erk, were assessed by western blot analysis following $24 \mathrm{~h}$ treatment with various concentrations of DAPT. A significant inhibitory effect on the expression of PI3K and $\beta$-catenin was observed, as well as on the phosphorylation of Akt, Src and p38MAPK, following treatment with 30 or $60 \mu \mathrm{mol} / 1$ DAPT (Fig. 3A-H). Although several studies have demonstrated the crosstalk between the Notch pathway and the Erk1/2 pathway (12), no effect on p-Erk1/2 levels was observed in any of the treatments (P>0.05; Fig. 3I and J).

\section{Discussion}

Among various molecular targets, Notch signaling is attractive as it is involved in numerous cellular processes, including cell fate specification, differentiation, proliferation and survival. 
A

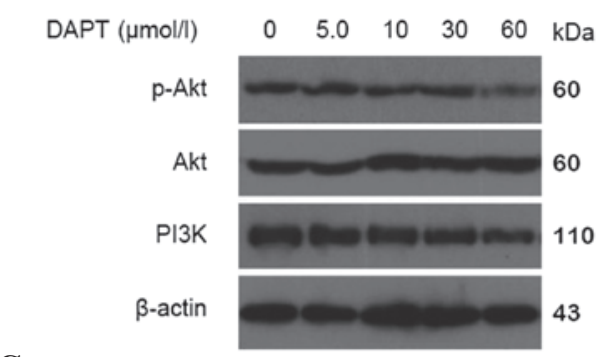

C

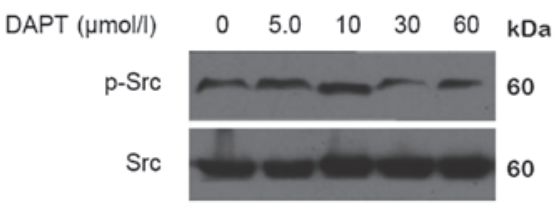

$\mathbf{E}$

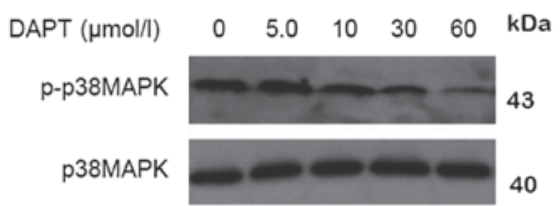

$\mathbf{G}$

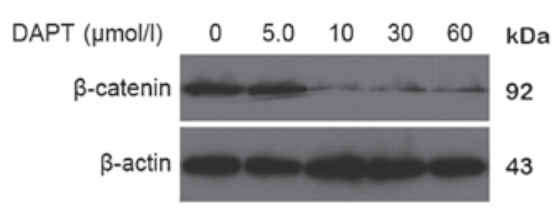

I

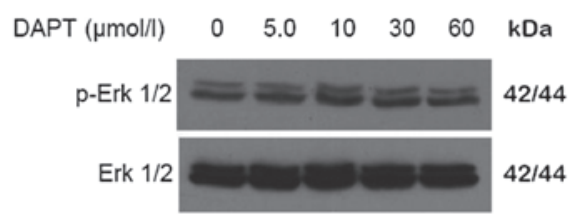

B

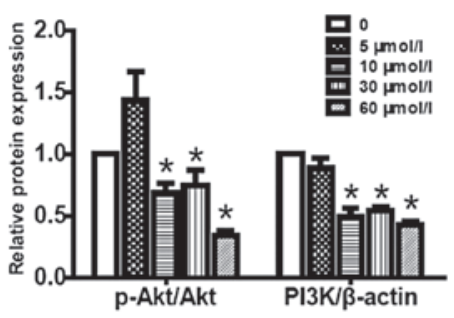

D

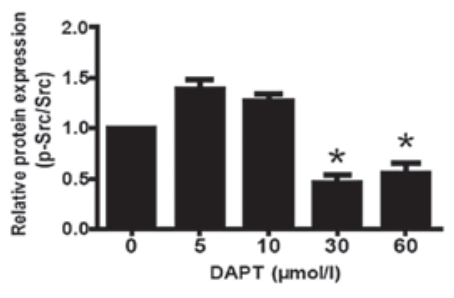

F

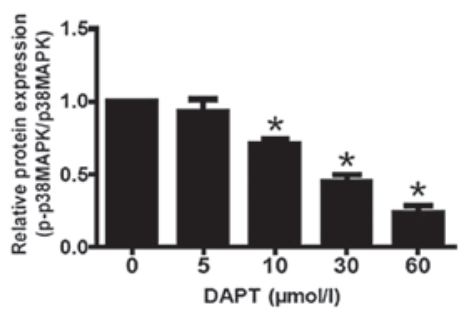

H

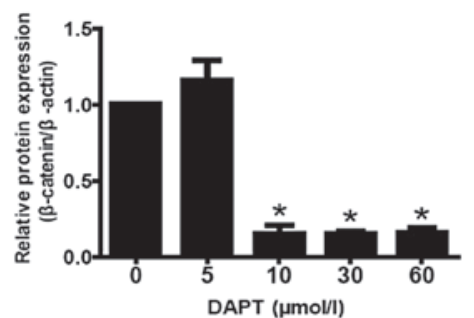

$\mathbf{J}$

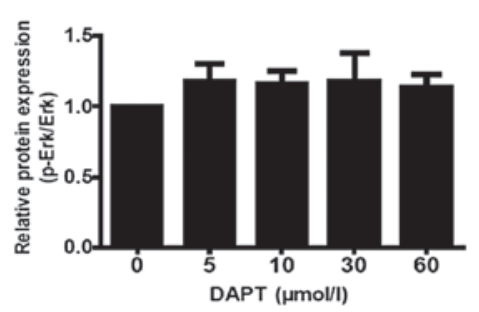

Figure 3. DAPT inhibits SO-Rb50 cell growth via co-operating with multiple pathways. Western blot analysis was performed to detect the activation of several classic pathways, including the (A and B) PI3K/Akt, (C and D) Src, (E and F) p38MAPK, (G and H) wnt/ $\beta$-catenin and (I and J) Erk1/2 pathways. Total protein expression levels of Akt, p38MAPK, Src and Erk1/2 were used as the internal control for their phosphorylated isoforms, respectively, and $\beta$-actin was set as the loading control for PI3K and $\beta$-catenin. Expression of PI3K and $\beta$-catenin, as well as phosphorylation of Akt, p38MAPK and Src, were significantly abated when treated with 30 or $60 \mu \mathrm{mol} / 1$ DAPT for $24 \mathrm{~h}$. (I and J) Of note, the total or phosphorylated Erk1/2 were not changed. DAPT, DAPT, $N$-[N-(3,5-difluorophenacetyl)-L-alanyl]-S-phenylglycine $t$-butyl ester; MAPK, mitogen activated protein kinase; PI3K, phosphoinositide 3-kinase; Erk, extracellular signal-regulated kinase. ${ }^{*} \mathrm{P}<0.05$.

Previous studies have demonstrated that Notch signaling is involved in the carcinogenesis, progression, invasion and neurovascular formation of numerous malignant tumors. Notch is able to function as an oncogene or a tumor suppressor, depending on the cell type and context. It acts as a tumor suppressor in skin tumors and non-small cell lung cancer $(13,14)$. However, it acts as an oncogene in renal, pancreatic, breast and prostate cancer (15). In the present study, Notch1 and Jagged2 were found to be highly expressed in the human retinoblastoma cell line. Inhibition of the Jagged2/Notch1 signaling pathway with DAPT suppressed cell growth via multiple crosstalk with other pathways. Therefore, the present study provided evidence supporting the role of Jagged2/Notch1 as oncogenes in human retinoblastoma cells.

Previous studies have demonstrated that Notch is aberrantly expressed in various types of human cancers, including 
breast, colon, cervical, lung, head and neck, renal cell carcinoma and pancreatic cancer (16). Typically, Notch1 is overexpressed in prostate cancer cell lines and human prostate cancer tissues (17). Furthermore, Notch1 expression levels in human prostate cancer tissues are elevated with increasing tumor grade (17). However, the role of the Notch pathway in retinoblastoma remains to be elucidated. Therefore, in the present study, the expression profiles of Notch ligands (Jagged1 and 2) and receptors (Notch1-3) were investigated for the first time, to the best of our knowledge. Notch1 and Jagged 2 were identified to be highly expressed in the human retinoblastoma cell line, which is similar to findings in myeloma cells, tongue carcinoma tissues and lung adenocarcinoma (18-20). These results further demonstrate that the expression of Notch signaling pathway proteins is frequently altered in malignancies.

Previous studies reported that components of the Notch signaling pathway are also the downstream targets of Notch signaling itself (21). In the present study, the expression levels of Jagged 2 and Notch1 were consistently and significantly suppressed when the cells were treated with various concentrations of DAPT. This suppressive effect was more profound on the ligand Jagged 2 and was dose-dependent. The effect of DAPT on the gene expression levels of Jagged1, Notch2 and Notch3 were not examined, as their expression was relatively low in SO-Rb50 cells.

Several studies have demonstrated that the downregulation of the Notch signaling pathway elicited a marked effect on the cell growth inhibition of cancer cells $(22,23)$. In the present study, when the Notch signaling pathway was inhibited with DAPT, SO-Rb50 cells underwent cell cycle arrest, which was in agreement with previous studies. The present study demonstrated that DAPT inhibited SO-Rb50 cell proliferation in a dose-dependent manner.

It is well accepted that numerous signaling pathways, including PI3K/Akt, Erk, p38MAPK, Src and wnt/ $\beta$-catenin signaling, are involved in tumor cell growth, invasion and metastasis. Since DAPT inhibited the proliferation of SO-Rb50 cells, the present study examined the effects of DAPT on the PI3K/Akt, Erk, p38MAPK, Src and wnt/ $\beta$-catenin signaling pathways.

The protein kinase Akt, which is able to induce $\gamma$-secretase, has been implicated in transducing Notch signaling (24). Notch1 activation enhanced melanoma cell survival and this effect was mediated by activation of the Akt pathway (25). Activating mutation of Notch1 comprises the most prominent genetic abnormality in T-cell acute lymphoblastic leukemia (T-ALL) in children. However, the majority of T-ALL cell lines with Notch1 mutations demonstrate chemoresistance to $\gamma$-secretase inhibitors (26). Previous studies revealed that PI3K/Akt and PTEN/AKT/mTOR pathways are involved in tumorigenesis (27). The present study examined whether the growth inhibition of SO-Rb50 cells was indeed due to the regulation of the Akt pathway. It was consistently found that the downregulation of the Notch signaling pathway by DAPT decreased Akt phosphorylation, suggesting that Akt inactivation may contribute to DAPT-induced cell growth arrest.

Src family kinases are non-receptor kinases overexpressed in the majority of pancreatic cancers and involved in cancer progression and metastasis. The expression of Src kinase is significantly higher in various types of invasive tumors $(28,29)$. In pancreatic cancer cells, Notch1 and c-Src proteins are physically associated, and this association mediates Notch1 processing and activation (30). Poorly differentiated tumors demonstrated a higher expression of Src kinase when compared with moderately- and well-differentiated tumors (31). Although Src kinase is moderately expressed in normal retinal tissues, Src kinase and phosphorylated signal transducer and activator of transcription 3 proteins in invasive retinoblastoma tumors are highly expressed, suggesting that they may be important in invasiveness by contributing to multiple aspects of tumor progression. In the present study, a high expression of $\mathrm{p}-\mathrm{Src}$ and Src in SO-Rb50 cells was detected, whereas DAPT inhibited the phosphorylation of Src without affecting the total Src expression. These results indicated that Src kinases are inactivated by DAPT.

The Wnt signaling pathway, which is involved in stem cell self-renewal and differentiation in various types of cancer, is a promising candidate pathway for cancer therapy (32). In chick and monkey retina, canonical Wnt signaling regulates the proliferation of retinal stem/progenitor cells, maintains them in an uncommitted state and suppresses neuronal differentiation. Although previous studies demonstrated that Wnt signaling activation significantly decreased the viability of retinoblastoma cells by inducing cell cycle arrest associated with the upregulation of p53 (33), sorted adenosine triphosphate-binding cassette sub-family $G$ member 2 (ABCG2)-positive stem-like cells exhibited higher levels of $\beta$-catenin than ABCG2-negative non-stem cells. This suggests that elevated canonical Wnt signaling regulates cancer stem-like cell populations in retinoblastoma. Therefore, inhibiting the canonical Wnt pathway may potentially suppress stem-like cell formation in retinoblastoma. $\beta$-catenin, which is involved in numerous protein-protein interactions (34), is considered the central hub of the Wnt signaling pathway. The present study found that DAPT also inhibited the expression of $\beta$-catenin in SO-Rb50 cells, which indicates an interaction between the Wnt and Notch signaling pathways.

Crosstalk among the intracellular signaling pathways is important in the determination of signaling specificity. In myogenesis, Notch was reported to specifically indirectly inactivate p38MAPK by inducing the expression of MKP-1, a member of the dual-specificity MAPK phosphatase (35). However, crosstalk between the Notch and the MAPK pathways in cancer has remained to be fully elucidated. In human nasopharyngeal carcinoma cells, a $\gamma$-secretase inhibitor suppressed human cell proliferation via inactivating Erk1/2 signaling pathways, without affecting p38MAPK signaling (22). In melanoma, Notch1 signaling promoted primary melanoma progression, partially mediated through the activation of the MAPK pathway (25). Notably, in the present study, examination of the Erk1/2 and p38MAPK levels in DAPT-treated SO-Rb50 cells revealed that p38MAPK decreased in a dose-dependent manner in response to DAPT, whereas the expression of $\mathrm{p}-\mathrm{Erk} 1 / 2$ was not affected. These data indicated that DAPT treatment resulted in the inhibition of the MAPK signaling pathway with specificity on p38MAPK signaling (however, not on Erk1/2 signaling). In addition, the crosstalk between Notch and other oncogenic pathways was likely to be context-dependent. 
In conclusion, the present study suggested that Jagged2/Notch1 signaling was involved in oncogenesis in retinoblastoma. Inhibition of the Notch pathway with DAPT inhibited the proliferation of human retinoblastoma cells via suppressing the PI3K/Akt, Src, p38MAPK and Wnt/ß-catenin signaling pathways.

\section{Acknowledgements}

The authors would like to thank the Department of Pathology, Zhongshan Ophthalmic Center for providing the human retinoblastoma cell line SO-Rb50.

\section{References}

1. Kivelä T: The epidemiological challenge of the most frequent eye cancer: retinoblastoma, an issue of birth and death Br J Ophthalmol 93: 1129-1131, 2009.

2. Rodriguez-Galindo C, Wilson MW, Chantada G, et al. Retinoblastoma: one world, one vision. Pediatrics 122: e763-e770, 2008.

3. Friend SH, Bernards R, Rogelj S, et al: A human DNA segment with properties of the gene that predisposes to retinoblastoma and osteosarcoma. Nature 323: 643-646, 1986.

4. Corson TW and Gallie BL: One hit, two hits, three hits, more? Genomic changes in the development of retinoblastoma. Genes Chromosomes Cancer 46: 617-634, 2007.

5. Dimaras H, Khetan V, Halliday W, et al: Loss of RB1 induces non-proliferative retinoma: increasing genomic instability correlates with progression to retinoblastoma. Hum Mol Genet 17 1363-1372, 2008.

6. Dyer MA and Bremner R: The search for the retinoblastoma cell of origin. Nat Rev Cancer 5: 91-101, 2005.

7. Kidd S, Lockett TJ and Young MW: The Notch locus of Drosophila melanogaster. Cell 34: 421-433, 1983.

8. Mullendore ME, Koorstra JB, Li YM, et al: Ligand-dependent Notch signaling is involved in tumor initiation and tumor maintenance in pancreatic cancer. Clin Cancer Res 15: 2291-2301, 2009.

9. Nam DH, Jeon HM, Kim S, et al: Activation of notch signaling in a xenograft model of brain metastasis. Clin Cancer Res 14: 4059-4066, 2008.

10. South AP, Cho RJ and Aster JC: The double-edged sword of Notch signaling in cancer. Semin Cell Dev Biol 23: 458-464, 2012.

11. Aguirre A, Rubio ME and Gallo V: Notch and EGFR pathway interaction regulates neural stem cell number and self-renewal. Nature 467: 323-327, 2010.

12. Zayzafoon M, Abdulkadir SA and McDonald JM: Notch signaling and ERK activation are important for the osteomimetic properties of prostate cancer bone metastatic cell lines. J Biol Chem 279: 3662-3670, 2004.

13. Ye YZ, Zhang ZH, Fan XY, et al: Notch3 overexpression associates with poor prognosis in human non-small-cell lung cancer. Med Oncol 30: 595, 2013.

14. Reichrath J and Reichrath S: Notch-signaling and nonmelanoma skin cancer: an ancient friend, revisited. Adv Exp Med Biol 727: 265-271, 2012.

15. Purow B: Notch inhibition as a promising new approach to cancer therapy. Adv Exp Med Biol 727: 305-319, 2012.

16. Miele L, Miao H and Nickoloff BJ: NOTCH signaling as a novel cancer therapeutic target. Curr Cancer Drug Targets 6: 313-323, 2006.
17. Bin Hafeez B, Adhami VM, Asim M, et al: Targeted knockdown of Notch1 inhibits invasion of human prostate cancer cells concomitant with inhibition of matrix metalloproteinase-9 and urokinase plasminogen activator. Clin Cancer Res 15: 452-459, 2009.

18. Chiron D, Maïga S, Descamps G, et al: Critical role of the NOTCH ligand JAG2 in self-renewal of myeloma cells. Blood Cells Mol Dis 48: 247-253, 2012.

19. Zhang TH, Liu HC, Zhu LJ, et al: Activation of Notch signaling in human tongue carcinoma. J Oral Pathol Med 40: 37-45, 2011.

20. Yang Y, Ahn YH, Gibbons DL, et al: The Notch ligand Jagged 2 promotes lung adenocarcinoma metastasis through a miR-200-dependent pathway in mice. J Clin Invest 121: 1373-1385, 2011.

21. Rodilla V, Villanueva A, Obrador-Hevia A, et al: Jagged1 is the pathological link between Wnt and Notch pathways in colorectal cancer. Proc Natl Acad Sci USA 106: 6315-6320, 2009.

22. Chen SM, Liu JP, Zhou JX, et al: Suppression of the notch signaling pathway by gamma-secretase inhibitor GSI inhibits human nasopharyngeal carcinoma cell proliferation. Cancer Lett 306: 76-84, 2011.

23. Shao H, Huang Q and Liu ZJ: Targeting Notch signaling for cancer therapeutic intervention. Adv Pharmacol 65: 191-234, 2012.

24. Guo D, Ye J, Dai J, et al: Notch-1 regulates Akt signaling pathway and the expression of cell cycle regulatory proteins cyclin D1, CDK2 and p21 in T-ALL cell lines. Leuk Res 33: 678-685, 2009.

25. Liu ZJ, Xiao M, Balint K, et al: Notch1 signaling promotes primary melanoma progression by activating mitogen-activated protein kinase/phosphatidylinositol 3-kinase-Akt pathways and up-regulating N-cadherin expression. Cancer Res 66: 4182-4190, 2006.

26. Mungamuri SK, Yang X, Thor AD and Somasundaram K: Survival signaling by Notch1: mammalian target of rapamycin (mTOR)-dependent inhibition of p53. Cancer Res 66: 4715-4724, 2006.

27. Guo D, Teng Q and Ji C: NOTCH and phosphatidylinositide 3-kinase/phosphatase and tensin homolog deleted on chromosome ten/AKT/mammalian target of rapamycin (mTOR) signaling in T-cell development and T-cell acute lymphoblastic leukemia. Leuk Lymphoma 52: 1200-1210, 2011.

28. Mohan A, Mallikarjuna K, Venkatesan N, Abhyankar D, Parikh PM and Krishnakumar S: The study of c-Src kinase and pStat3 protein expression in retinoblastoma. Exp Eye Res 83: 736-740, 2006.

29. Ishizawar R and Parsons SJ: c-Src and cooperating partners in human cancer. Cancer Cell 6: 209-214, 2004.

30. Ma YC, Shi C, Zhang YN, et al: The tyrosine kinase c-Src directly mediates growth factor-induced Notch-1 and Furin interaction and Notch-1 activation in pancreatic cancer cells. PLoS One 7: e33414, 2012.

31. Chen YS, Wu MJ, Huang CY, Lin SC, Chuang TH, Yu CC and Lo JF: CD133/Src axis mediates tumor initiating property and epithelial-mesenchymal transition of head and neck cancer. PLoS One 6: e28053,2011.

32. Reya $\mathrm{T}$ and Clevers $\mathrm{H}$ : Wnt signalling in stem cells and cancer. Nature 434: 843-850, 2005.

33. Tell S, Yi H, Jockovich ME, Murray TG and Hackam AS: The Wnt signaling pathway has tumor suppressor properties in retinoblastoma. Biochem Biophys Res Commun 349: 261-269, 2006.

34. Hahne G and Grossmann TN: Direct targeting of beta-catenin: Inhibition of protein-protein interactions for the inactivation of Wnt signaling. Bioorg Med Chem 21: 4020-4026, 2013.

35. Kondoh K, Sunadome K and Nishida E: Notch signaling suppresses p38 MAPK activity via induction of MKP-1 in myogenesis. J Biol Chem 282: 3058-3065, 2007. 